\title{
The Interval-Valued Triangular Fuzzy Soft Set and Its Method of Dynamic Decision Making
}

\author{
Xiaoguo Chen, ${ }^{1,2}$ Hong Du, ${ }^{2}$ and Yue Yang ${ }^{1}$ \\ ${ }^{1}$ School of Mechanics and Civil Engineering, China University of Mining \& Technology, Beijing 100083, China \\ ${ }^{2}$ School of Science, Heilongjiang University of Science and Technology, Harbin 150022, China \\ Correspondence should be addressed to Xiaoguo Chen; kjdxcxg@sohu.com
}

Received 26 May 2014; Revised 12 August 2014; Accepted 12 August 2014; Published 26 August 2014

Academic Editor: Shuming Wang

Copyright (c) 2014 Xiaoguo Chen et al. This is an open access article distributed under the Creative Commons Attribution License, which permits unrestricted use, distribution, and reproduction in any medium, provided the original work is properly cited.

\begin{abstract}
A concept of interval-valued triangular fuzzy soft set is presented, and some operations of "AND," "OR," intersection, union and complement, and so forth are defined. Then some relative properties are discussed and several conclusions are drawn. A dynamic decision making model is built based on the definition of interval-valued triangular fuzzy soft set, in which period weight is determined by the exponential decay method. The arithmetic weighted average operator of interval-valued triangular fuzzy soft set is given by the aggregating thought, thereby aggregating interval-valued triangular fuzzy soft sets of different time-series into a collective interval-valued triangular fuzzy soft set. The formulas of selection and decision values of different objects are given; therefore the optimal decision making is achieved according to the decision values. Finally, the steps of this method are concluded, and one example is given to explain the application of the method.
\end{abstract}

\section{Introduction}

Complex problems involving vagueness and uncertainty are pervasive in many areas of modern technology. These practical problems arise in diverse areas such as economics, engineering, environmental science, and social science [1]. While a wide variety of mathematical disciplines like probability theory, fuzzy set theory [2], rough set theory [3], and interval mathematics [4] are well known and frequently employed as useful mathematical approaches to modeling, each of them has its advantages as well as inherent limitations. One major weakness of these theories may be the inadequacy of the parameterization tool in them [5]. Consequently, Molodtsov initiated the concept of soft theory as a mathematical tool, which is free from the above difficulties for dealing with uncertainties [5].

Soft set theory has received much attention and in-depth study since its introduction. Maji et al. [6] presented the concept of the fuzzy soft set which is based on a combination of the fuzzy set and soft set models. In the same year, Maji et al. [7] presented the notion of the intuitionistic fuzzy soft set theory which is based on a combination of the intuitionistic fuzzy set and soft set models. Yang et al. [8] proposed the concept of the interval-valued fuzzy soft set by combining the interval-valued fuzzy set and soft set models. Jiang et al. [9] presented the theory of interval-valued intuitionistic fuzzy soft set by combining the interval-valued intuitionistic fuzzy set and soft set. Feng et al. [10] made a primary discussion of soft set through combining the rough set and fuzzy set. Ali [11] raised the concept of rough soft set by combining rough set and soft set. $\mathrm{Xu}$ et al. [12] used vague set [13] to make an extension of soft set and proposed vague soft set theory. Majumdar and Samanta [14] proposed the generalized concept of fuzzy soft set theory. On the basis of previous studies, many researchers have carried out particular exploration on the operations of intersection, union, complement, and subset among various soft sets [15-21]. Xiao et al. [22] employed fuzzy soft set into the application of economic forecasting. Maji and Roy firstly introduced the soft set and fuzzy soft set into decision making problem and employed the rough set theory in attribute reduction [23, 24], but the decision making method in [24] pretended some errors which had been discussed by Kong et al. [25]. Çağman and Enginoğlu [26] redefined the concept of soft set proposed 
by Molodtsov and discussed its relevant algorithm. In the meantime, they also defined decision function and applied it into the decision making problem. Çağman and Enginoğ [27] defined soft matrix on the basis of soft set, discussed its relevant properties and operation, and constructed the maximum and minimum soft functions to solve practical decision making problems. Feng et al. [28] and Jiang et al. [29] carried out further study of soft set, respectively, and proposed the threshold vector for constructing the level soft set in decision making, converted different types of soft sets into classic soft sets, and then obtained the optimal decision by the opportunity value of different objects. Based on [28, 29], Mao et al. [30] proposed a decision making method on the basis of timing fuzzy soft set. Kuang and Xiao [31, 32] raised the definition of introduced triangular fuzzy soft set and trapezoidal fuzzy soft set, introduced the relevant operating properties of them, and built the corresponding decision making models.

Despite the fact that the studies above have widely discussed the extension of the soft set, there is relatively little literature on the study of the interval-valued triangle fuzzy soft set. Besides, the studies on decision above are almost static, while practical problems always need dynamic analysis, so it is essential to introduce the time variate into the decision making of the fuzzy soft set. Based on this, definition of the interval-valued triangle fuzzy soft set is presented and relevant operational properties are discussed in this paper. The paper establishes the decision making model of the dynamic interval-valued triangle fuzzy soft set which considers the time variate and whose time weights can be determined by exponential decay method of [33]. Using the thought of integration [34], the paper defines the arithmetic weighted average operator of interval-valued triangle fuzzy soft set and gives methods to integrate the dynamic intervalvalued triangle fuzzy soft set. In this paper, the decision methods of $[8,31]$ are used in combination to calculate a solution formula of the selection values and decision values based on the presented interval-valued triangle fuzzy soft set. Then the drawn solution formula is applied to decision analysis.

The purpose of this paper is to combine the intervalvalued triangular fuzzy set and soft set, from which we can obtain a new soft set model: interval-valued triangular fuzzy soft set. To facilitate our discussion, we first introduce the standard soft set, fuzzy soft set, interval-valued fuzzy soft set, and triangular fuzzy soft set in Section 2. The concept of interval-valued triangular fuzzy soft set is presented in Section 3. Then the "AND," "OR," intersection, union, and complement operations on the interval-valued triangular fuzzy soft sets are defined. In Section 4, the interval-valued triangular fuzzy soft set is used to analyze a decision making problem. Then a specific example is analyzed and solved with this technique in Section 5. At last, we draw conclusions in Section 6.

\section{Preliminary}

In this section, we briefly review the concepts of soft set, fuzzy soft set, interval-valued fuzzy soft set, and triangular fuzzy soft set. Further details could be found in [5-9]. Molodtsov [5] defined the soft set in the following way. Let $U$ be an initial universe of objects and let $E$ be the set of parameters in relation to objects in $U$. Parameters are usually attributes, characteristics, or properties of objects. Let $P(U)$ denote the power set of $U$ and $A \subseteq E$.

Definition 1 (see [5]). A pair $(F, A)$ is called a soft set over $U$, where $F$ is a mapping given by $F: A \rightarrow P(U)$. In other words, the soft set is not a kind of set but a parameterized family of subsets of the set $U$. For any parameter $e \in A, F(e) \subseteq$ $U$ may be considered as the set of $e$ approximate elements of the soft set $(F, A)$.

Definition 2 (see [6]). Let $\Gamma(U)$ be the set of all fuzzy subsets of $U$. Let $E$ be a set of parameters and $A \subseteq E$. A pair $(F, A)$ is called a fuzzy soft set over $U$, where $F$ is a mapping given by $F: A \rightarrow \Gamma(U)$.

Let us denote $\mu_{F(e)}(x)$ by the membership degree that object $x$ holds parameter $e$ where $x \in U$ and $e \in A$; then $F(e)$ can be written as a fuzzy set such that $F(e)=\left\{\left\langle x, \mu_{F(e)}(x)\right\rangle \mid\right.$ $x \in U\}$.

In the following, we will introduce the notion of intervalvalued fuzzy soft set. Firstly, let us briefly introduce the concept of the interval-valued fuzzy set of [8].

An interval-valued fuzzy set $X$ on a universe $U$ is a mapping such that $X: U \rightarrow \operatorname{Int}([0,1])$, where $\operatorname{Int}([0,1])$ stands for the set of all closed subintervals of $[0,1]$; the set of all interval-valued fuzzy sets on $U$ is denoted by $\operatorname{IVF}(U)$. Suppose that $X \in \operatorname{IVF}(U), x \in U, \mu_{X}(x)=\left[\mu_{X}^{-}(x), \mu_{X}^{+}(x)\right]$ is called the degree of membership of an element $x$ to $X . \mu_{X}^{-}(x)$ and $\mu_{X}^{+}(x)$ are referred to as the lower and upper degrees of membership of $x$ to $X$, where $0 \leq \mu_{X}^{-}(x) \leq \mu_{X}^{+}(x) \leq 1$.

Definition 3 (see [8]). Let $U$ be an initial universe and let $E$ be a set of parameters. $\operatorname{IVF}(U)$ denotes the set of all intervalvalued fuzzy sets of $U$. Let $A \subseteq E$. A pair $(F, A)$ is an intervalvalued fuzzy soft set over $U$, where $F$ is a mapping given by $F: A \rightarrow \operatorname{IVF}(U)$.

Let us denote $\mu_{F(e)}(x)=\left[\mu_{F(e)}^{-}(x), \mu_{F(e)}^{+}(x)\right]$ by the membership degree that object $x$ holds parameter $e$ where $x \in U$ and $e \in A$; then $F(e)$ can be written as an interval-valued fuzzy set as

$$
F(e)=\left\{\left\langle x,\left[\mu_{F(e)}^{-}(x), \mu_{F(e)}^{+}(x)\right]\right\rangle \mid x \in U\right\} .
$$

Definition 4 (see [31]). Let $U$ be an initial universe and let $E$ be a set of parameters. $\Gamma(U)$ denotes the set of all triangular fuzzy sets of $U$. Let $A \subseteq E$. A pair $(\widetilde{\Im}, A)$ is called a triangle-valued fuzzy soft set over $U$, where $\widetilde{\mathfrak{I}}$ is a mapping given by $\widetilde{\mathfrak{J}}: A \rightarrow$ $\Gamma(U)$.

A triangle-valued fuzzy soft set is a parameterized family of triangle-valued fuzzy subsets of $U$; thus its universe is the set of all triangle-valued fuzzy sets of $U$, that is, $\Gamma(U)$. Obviously, a triangle-valued fuzzy soft set is a special case of a fuzzy soft set. 


\section{Interval-Valued Triangular Fuzzy Soft Sets}

Definition 5 (see [35]). If

$$
s=\left\{\begin{array}{l}
\left(a^{-}, b, c^{-}\right) \\
\left(a^{+}, b, c^{+}\right),
\end{array}\right.
$$

where $0<a^{-} \leq a^{+} \leq b \leq c^{-} \leq c^{+}$, then $s$ is called an intervalvalued triangular fuzzy number and also is denoted as $s=$ $\left[\left(a^{-}, a^{+}\right) ; b ;\left(c^{-}, c^{+}\right)\right]$.

If $0<a^{-} \leq a^{+} \leq b \leq c^{-} \leq c^{+} \leq 1$, then $s$ is called a standard interval-valued triangular fuzzy number. The following interval-valued triangular fuzzy numbers described in this paper stand for standard interval-valued triangular fuzzy numbers.

Definition 6. Suppose $s=\left[\left(a^{-}, a^{+}\right) ; b ;\left(c^{-}, c^{+}\right)\right]$and $t=\left[\left(m^{-}\right.\right.$, $\left.\left.m^{+}\right) ; h ;\left(k^{-}, k^{+}\right)\right]$are interval-valued triangular fuzzy numbers; if $a^{-} \leq m^{-}, a^{+} \leq m^{+}, b \leq h, c^{-} \leq k^{-}$, and $c^{+} \leq k^{+}$, it is said $s \leq t$.

Definition 7. Suppose $s=\left[\left(a^{-}, a^{+}\right) ; b ;\left(c^{-}, c^{+}\right)\right]$and $t=\left[\left(m^{-}\right.\right.$, $\left.\left.m^{+}\right) ; h ;\left(k^{-}, k^{+}\right)\right]$are interval-valued triangular fuzzy numbers; then we have

$$
\begin{aligned}
& \text { (1) } s \cap t=\left[\left(\inf \left(a^{-}, m^{-}\right), \inf \left(a^{+}, m^{+}\right)\right)\right. \text {; } \\
& \left.\inf (b, h) ;\left(\inf \left(c^{-}, k^{-}\right), \inf \left(c^{+}, k^{+}\right)\right)\right], \\
& \text {(2) } s \cup t=\left[\left(\sup \left(a^{-}, m^{-}\right), \sup \left(a^{+}, m^{+}\right)\right)\right. \text {; } \\
& \left.\sup (b, h) ;\left(\sup \left(c^{-}, k^{-}\right), \sup \left(c^{+}, k^{+}\right)\right)\right] .
\end{aligned}
$$

Definition 8. Suppose $t=\left[\left(\mathrm{m}^{-}, \mathrm{m}^{+}\right) ; h ;\left(k^{-}, k^{+}\right)\right]$is an interval-valued triangular fuzzy number; then the complementary set of $t$ is denoted as

$$
t^{c}=\left[\left(1-k^{+}, 1-k^{-}\right) ; 1-h ;\left(1-m^{+}, 1-m^{-}\right)\right] .
$$

Definition 9. Suppose $s=\left[\left(a^{-}, a^{+}\right) ; b ;\left(c^{-}, c^{+}\right)\right]$and $t=\left[\left(m^{-}\right.\right.$, $\left.\left.m^{+}\right) ; h ;\left(k^{-}, k^{+}\right)\right]$are interval-valued triangular fuzzy numbers; for the constant $k>0$, then we have

$$
\text { (1) } \begin{gathered}
s+t=\left[\left(\left(a^{-}+m^{-}-a^{-} m^{-}\right),\left(a^{+}+m^{+}-a^{+} m^{+}\right)\right) ;\right. \\
+h-b h ;\left(\left(c^{-}+k^{-}-c^{-} k^{-}\right),\right. \\
\left.\left.\left(c^{+}+k^{+}-c^{+} k^{+}\right)\right)\right],
\end{gathered}
$$

(2) $k s=\left[\left(1-\left(1-a^{-}\right)^{k}, 1-\left(1-a^{+}\right)^{k}\right)\right.$;

$$
\left.1-(1-b)^{k} ;\left(1-\left(1-c^{-}\right)^{k}, 1-\left(1-c^{+}\right)^{k}\right)\right] .
$$

Definition 10. Let $U$ be a universe and let $E$ be a set of parameters. Suppose that $\Gamma(U)$ stands for all the interval-valued triangular fuzzy sets over $U, A \subseteq E$; then we say that $(F, A)$ is an interval-valued triangular fuzzy soft subset of $U$, where mapping $F: A \rightarrow \Gamma(U)$. For $\forall e \in A$,

$$
F(e)=\left\{\left\langle x, s_{F(e)}(x)\right\rangle \mid x \in U\right\},
$$

where $s_{F(e)}(x)$ is the interval-valued triangular fuzzy number of $x$ in $F(e)$.
Example 11. Suppose the following: $U$ is the set of the houses under consideration and

$$
U=\left\{x_{1}, x_{2}, x_{3}, x_{4}, x_{5}, x_{6}\right\} .
$$

$A$ is the set of parameters and $A=\left\{e_{1}, e_{2}, e_{3}, e_{4}\right\}=$ beautiful, wooden, cheap, in the green surroundings $\}$.

The tabular representation of an interval-valued triangular fuzzy soft set $(F, A)$ is shown in Table 1 . Obviously, the precise evaluation for each object on each parameter is unknown, while the interval-valued triangular fuzzy set of such an evaluation is given. The interval-valued triangular fuzzy soft set $(F, A)$ is given as $(F, A)=\left\{F\left(e_{1}\right), F\left(e_{2}\right), F\left(e_{3}\right), F\left(e_{4}\right)\right\}$, where

$$
\begin{aligned}
F\left(e_{1}\right)=\{ & \left\langle x_{1},[(0.3,0.4) ; 0.5 ;(0.5,0.6)]\right\rangle, \\
& \left\langle x_{2},[(0.5,0.6) ; 0.7 ;(0.8,0.9)]\right\rangle, \\
& \left\langle x_{3},[(0.4,0.5) ; 0.6 ;(0.6,0.7)]\right\rangle, \\
& \left\langle x_{4},[(0.5,0.6) ; 0.6 ;(0.7,0.8)]\right\rangle, \\
& \left\langle x_{5},[(0.2,0.4) ; 0.5 ;(0.5,0.6)]\right\rangle, \\
& \left.\left\langle x_{6},[(0.6,0.7) ; 0.7 ;(0.7,0.8)]\right\rangle\right\}, \\
F\left(e_{2}\right)=\{ & \left\langle x_{1},[(0.5,0.6) ; 0.7 ;(0.7,0.8)]\right\rangle,
\end{aligned}
$$

$$
\begin{gathered}
\left\langle x_{2},[(0.4,0.5) ; 0.6 ;(0.6,0.7)]\right\rangle, \\
\left\langle x_{3},[(0.7,0.8) ; 0.8 ;(0.8,0.9)]\right\rangle, \\
\left\langle x_{4},[(0.6,0.7) ; 0.7 ;(0.7,0.8)]\right\rangle, \\
\left\langle x_{5},[(0.2,0.3) ; 0.6 ;(0.6,0.7)]\right\rangle, \\
\left.\left\langle x_{6},[(0.3,0.4) ; 0.5 ;(0.5,0.6)]\right\rangle\right\},
\end{gathered}
$$$$
F\left(e_{3}\right)=\left\{\left\langle x_{1},[(0.3,0.4) ; 0.5 ;(0.6,0.7)]\right\rangle,\right.
$$$$
\left\langle x_{2},[(0.4,0.5) ; 0.5 ;(0.5,0.6)]\right\rangle \text {, }
$$$$
\left\langle x_{3},[(0.6,0.7) ; 0.7 ;(0.8,0.9)]\right\rangle \text {, }
$$$$
\left\langle x_{4},[(0.5,0.6) ; 0.6 ;(0.7,0.8)]\right\rangle \text {, }
$$$$
\left\langle x_{5},[(0.7,0.8) ; 0.8 ;(0.8,0.9)]\right\rangle \text {, }
$$$$
\left.\left\langle x_{6},[(0.5,0.6) ; 0.7 ;(0.7,0.8)]\right\rangle\right\},
$$$$
F\left(e_{4}\right)=\left\{\left\langle x_{1},[(0.2,0.3) ; 0.4 ;(0.5,0.6)]\right\rangle,\right.
$$$$
\left\langle x_{2},[(0.3,0.4) ; 0.5 ;(0.6,0.8)]\right\rangle \text {, }
$$$$
\left\langle x_{3},[(0.1,0.2) ; 0.3 ;(0.4,0.5)]\right\rangle \text {, }
$$$$
\left\langle x_{4},[(0.3,0.4) ; 0.5 ;(0.5,0.7)]\right\rangle \text {, }
$$$$
\left\langle x_{5},[(0.4,0.5) ; 0.5 ;(0.5,0.6)]\right\rangle \text {, }
$$$$
\left.\left\langle x_{6},[(0.3,0.4) ; 0.5 ;(0.6,0.7)]\right\rangle\right\} \text {. }
$$ 
Definition 12. Assuming $(F, A)$ and $(G, B)$ are the intervalvalued triangular fuzzy soft sets on the universe $U$, then if and only if $A \subseteq B$ and $\forall e \in A, F(e)$ is the interval-valued triangular fuzzy subset of $G(e)$. For $\forall x \in U, x \in F(e)$, and $x \in$ $G(e)$, the interval-valued triangular fuzzy numbers $s_{F(e)}(x)$ and $s_{G(e)}(x)$ satisfy the condition $s_{F(e)}(x) \leq s_{G(e)}(x)$; then we say that $(F, A)$ is the interval-valued triangular fuzzy soft subset of $(G, B)$, and it is written as $F(A) \widetilde{\subseteq} G(B)$. If $(G, B)$ is the interval-valued triangular fuzzy soft subset of $(F, A)$, then $(F, A)$ is the interval-valued triangular fuzzy soft superset of $(G, B)$.

Example 13. Given two interval-valued triangular fuzzy soft sets $(F, A)$ and $(G, B)$ and a set of houses under consideration $U=\left\{x_{1}, x_{2}, x_{3}, x_{4}, x_{5}, x_{6}\right\}$, where

$$
A=\left\{e_{1}, e_{2}\right\}=\{\text { beautiful, wooden }\},
$$

$B=\left\{e_{1}, e_{2}, e_{3}\right\}=\{$ beautiful, wooden, cheap $\}$,

$$
\begin{aligned}
F\left(e_{1}\right)=\{ & \left\langle x_{1},[(0.3,0.4) ; 0.5 ;(0.5,0.6)]\right\rangle, \\
& \left\langle x_{2},[(0.5,0.6) ; 0.7 ;(0.8,0.9)]\right\rangle, \\
& \left\langle x_{3},[(0.4,0.5) ; 0.6 ;(0.6,0.7)]\right\rangle, \\
& \left\langle x_{4},[(0.5,0.6) ; 0.6 ;(0.7,0.8)]\right\rangle, \\
& \left\langle x_{5},[(0.2,0.4) ; 0.5 ;(0.5,0.6)]\right\rangle, \\
& \left.\left\langle x_{6},[(0.6,0.7) ; 0.7 ;(0.7,0.8)]\right\rangle\right\},
\end{aligned}
$$$$
F\left(e_{2}\right)=\left\{\left\langle x_{1},[(0.5,0.6) ; 0.7 ;(0.7,0.8)]\right\rangle,\right.
$$$$
\left\langle x_{2},[(0.4,0.5) ; 0.6 ;(0.6,0.7)]\right\rangle,
$$$$
\left\langle x_{3},[(0.7,0.8) ; 0.8 ;(0.8,0.9)]\right\rangle,
$$$$
\left\langle x_{4},[(0.6,0.7) ; 0.7 ;(0.7,0.8)]\right\rangle,
$$$$
\left\langle x_{5},[(0.2,0.3) ; 0.6 ;(0.6,0.7)]\right\rangle,
$$$$
\left.\left\langle x_{6},[(0.3,0.4) ; 0.5 ;(0.5,0.6)]\right\rangle\right\},
$$$$
G\left(e_{1}\right)=\left\{\left\langle x_{1},[(0.4,0.5) ; 0.5 ;(0.6,0.7)]\right\rangle,\right.
$$$$
\left\langle x_{2},[(0.6,0.7) ; 0.8 ;(0.8,0.9)]\right\rangle,
$$$$
\left\langle x_{3},[(0.5,0.6) ; 0.6 ;(0.7,0.8)]\right\rangle \text {, }
$$$$
\left\langle x_{4},[(0.6,0.7) ; 0.7 ;(0.8,0.9)]\right\rangle \text {, }
$$$$
\left\langle x_{5},[(0.3,0.4) ; 0.5 ;(0.6,0.7)]\right\rangle \text {, }
$$$$
\left.\left\langle x_{6},[(0.6,0.7) ; 0.8 ;(0.8,0.9)]\right\rangle\right\},
$$

$$
\begin{aligned}
G\left(e_{2}\right)=\{ & \left\langle x_{1},[(0.6,0.7) ; 0.7 ;(0.8,0.9)]\right\rangle, \\
& \left\langle x_{2},[(0.4,0.5) ; 0.6 ;(0.7,0.8)]\right\rangle, \\
& \left\langle x_{3},[(0.7,0.8) ; 0.9 ;(0.9,0.9)]\right\rangle, \\
& \left\langle x_{4},[(0.7,0.8) ; 0.8 ;(0.8,0.9)]\right\rangle,
\end{aligned}
$$

$$
\begin{aligned}
\left\langle x_{5},[(0.3,0.4) ; 0.6 ;(0.6,0.7)]\right\rangle, & \left.\left\langle x_{6},[(0.4,0.5) ; 0.5 ;(0.6,0.7)]\right\rangle\right\}, \\
G\left(e_{3}\right)= & \left\langle\left\langle x_{1},[(0.4,0.5) ; 0.5 ;(0.6,0.7)]\right\rangle,\right. \\
& \left\langle x_{2},[(0.4,0.5) ; 0.6 ;(0.6,0.7)]\right\rangle, \\
& \left\langle x_{3},[(0.6,0.7) ; 0.8 ;(0.8,0.9)]\right\rangle, \\
& \left\langle x_{4},[(0.5,0.6) ; 0.7 ;(0.8,0.9)]\right\rangle, \\
& \left\langle x_{5},[(0.4,0.5) ; 0.5 ;(0.6,0.7)]\right\rangle, \\
& \left.\left\langle x_{6},[(0.2,0.3) ; 0.4 ;(0.4,0.5)]\right\rangle\right\}
\end{aligned}
$$

then, apparently, we have $F(A) \widetilde{\subseteq} G(B)$.

Definition 14. Let $(F, A)$ and $(G, B)$ be two interval-valued triangular fuzzy soft sets over universe $U$; if $F(A) \widetilde{\subseteq} G(B)$ and $G(B) \widetilde{\subseteq} F(A)$, then we say that $(F, A)$ and $(G, B)$ are equal, and it can be written as $F(A) \cong G(B)$.

Definition 15. Suppose $(F, A)$ is an interval-valued triangular fuzzy soft set over universe $U ;(F, A)^{c}=\left(F^{c}, A\right)$ is called the complement of $(F, A)$, where $F^{c}: A \rightarrow \Gamma(U)$. For $\forall e \in A$,

$$
F^{c}(e)=(F(e))^{c}=\left\{\left\langle x, s_{F(e)}^{c}(x)\right\rangle \mid x \in U\right\},
$$

where $s_{F(e)}(x)$ is the interval-valued triangular fuzzy number of $x$ in $F(e)$.

Obviously, we have $\left((F, A)^{c}\right)^{c}=(F, A)$ according to Definition 15.

Example 16. Considering another interval-valued triangular fuzzy soft set $(G, B)$ shown in Table 2 , the universe $U$ is the same as the universe in Table 1 , while the set of the parameters

$$
\begin{aligned}
B & =\left\{\varepsilon_{1}, \varepsilon_{2}, \varepsilon_{3}\right\} \\
& =\{\text { convenient traffic, modern style, in good repair }\} ;
\end{aligned}
$$

thus we have the following formulas according to Definition 15:

$$
\begin{aligned}
& G^{c}\left(\varepsilon_{1}\right)=\{\left\langle x_{1},[(0.5,0.6) ; 0.7 ;(0.7,0.8)]\right\rangle, \\
&\left\langle x_{2},[(0.1,0.2) ; 0.2 ;(0.3,0.4)]\right\rangle, \\
&\left\langle x_{3},[(0.3,0.4) ; 0.5 ;(0.5,0.6)]\right\rangle, \\
&\left\langle x_{4},[(0.1,0.2) ; 0.3 ;(0.4,0.5)]\right\rangle, \\
&\left\langle x_{5},[(0.4,0.5) ; 0.5 ;(0.6,0.7)]\right\rangle, \\
&\left.\left\langle x_{6},[(0.2,0.3) ; 0.3 ;(0.3,0.5)]\right\rangle\right\}, \\
& G^{c}\left(\varepsilon_{2}\right)=\left\{\left\langle x_{1},[(0.2,0.3) ; 0.4 ;(0.5,0.6)]\right\rangle,\right. \\
&\left\langle x_{2},[(0.1,0.2) ; 0.3 ;(0.4,0.5)]\right\rangle, \\
&\left\langle x_{3},[(0.3,0.4) ; 0.4 ;(0.6,0.7)]\right\rangle,
\end{aligned}
$$


TABLE 1: An interval-valued triangular fuzzy soft set $(F, A)$.

\begin{tabular}{ccccc}
\hline$U$ & $e_{1}$ & $e_{2}$ & $e_{3}$ & $e_{4}$ \\
\hline$x_{1}$ & {$[(0.3,0.4) ; 0.5 ;(0.5,0.6)]$} & {$[(0.5,0.6) ; 0.7 ;(0.7,0.8)]$} & {$[(0.3,0.4) ; 0.5 ;(0.6,0.7)]$} & {$[(0.2,0.3) ; 0.4 ;(0.5,0.6)]$} \\
$x_{2}$ & {$[(0.5,0.6) ; 0.7 ;(0.8,0.9)]$} & {$[(0.4,0.5) ; 0.6 ;(0.6,0.7)]$} & {$[(0.4,0.5) ; 0.5 ;(0.5,0.6)]$} & {$[(0.3,0.4) ; 0.5 ;(0.6,0.8)]$} \\
$x_{3}$ & {$[(0.4,0.5) ; 0.6 ;(0.6,0.7)]$} & {$[(0.7,0.8) ; 0.8 ;(0.8,0.9)]$} & {$[(0.6,0.7) ; 0.7 ;(0.8,0.9)]$} & {$[(0.1,0.2) ; 0.3 ;(0.4,0.5)]$} \\
$x_{4}$ & {$[(0.5,0.6) ; 0.6 ;(0.7,0.8)]$} & {$[(0.6,0.7) ; 0.7 ;(0.7,0.8)]$} & {$[(0.5,0.6) ; 0.6 ;(0.7,0.8)]$} & {$[(0.3,0.4) ; 0.5 ;(0.5,0.7)]$} \\
$x_{5}$ & {$[(0.2,0.4) ; 0.5 ;(0.5,0.6)]$} & {$[(0.2,0.3) ; 0.6 ;(0.6,0.7)]$} & {$[(0.7,0.8) ; 0.8 ;(0.8,0.9)]$} & {$[(0.4,0.5) ; 0.5 ;(0.5,0.6)]$} \\
$x_{6}$ & {$[(0.6,0.7) ; 0.7 ;(0.7,0.8)]$} & {$[(0.3,0.4) ; 0.5 ;(0.5,0.6)]$} & {$[(0.5,0.6) ; 0.7 ;(0.7,0.8)]$} & {$[(0.3,0.4) ; 0.5 ;(0.6,0.7)]$} \\
\hline
\end{tabular}

TABLE 2: An interval-valued triangular fuzzy soft set $(G, B)$.

\begin{tabular}{lccr}
\hline$U$ & $\varepsilon_{1}$ & $\varepsilon_{2}$ & $\varepsilon_{3}$ \\
\hline$x_{1}$ & {$[(0.2,0.3) ; 0.3 ;(0.4,0.5)]$} & {$[(0.4,0.5) ; 0.6 ;(0.7,0.8)]$} & {$[(0.3,0.5) ; 0.6 ;(0.6,0.7)]$} \\
$x_{2}$ & {$[(0.6,0.7) ; 0.8 ;(0.8,0.9)]$} & {$[(0.5,0.6) ; 0.7 ;(0.8,0.9)]$} & {$[(0.4,0.5) ; 0.6 ;(0.7,0.8)]$} \\
$x_{3}$ & {$[(0.4,0.5) ; 0.5 ;(0.6,0.7)]$} & {$[(0.3,0.4) ; 0.6 ;(0.6,0.7)]$} & {$[(0.2,0.3) ; 0.4 ;(0.4,0.5)]$} \\
$x_{4}$ & {$[(0.5,0.6) ; 0.7 ;(0.8,0.9)]$} & {$[(0.5,0.6) ; 0.6 ;(0.7,0.8)]$} & {$[(0.3,0.4) ; 0.5 ;(0.5,0.6)]$} \\
$x_{5}$ & {$[(0.3,0.4) ; 0.5 ;(0.5,0.6)]$} & {$[(0.3,0.4) ; 0.4 ;(0.4,0.5)]$} & {$[(0.5,0.6) ; 0.7 ;(0.7,0.8)]$} \\
$x_{6}$ & {$[(0.5,0.7) ; 0.7 ;(0.7,0.8)]$} & {$[(0.2,0.4) ; 0.5 ;(0.6,0.7)]$} & {$[(0.6,0.7) ; 0.8 ;(0.8,0.9)]$} \\
\hline
\end{tabular}

$$
\begin{aligned}
&\left\langle x_{4},[(0.2,0.3) ; 0.4 ;(0.4,0.5)]\right\rangle, \\
&\left\langle x_{5},[(0.5,0.6) ; 0.6 ;(0.6,0.7)]\right\rangle, \\
&\left.\left\langle x_{6},[(0.3,0.4) ; 0.5 ;(0.6,0.8)]\right\rangle\right\}, \\
& G^{c}\left(\varepsilon_{3}\right)=\left\{\left\langle x_{1},[(0.3,0.4) ; 0.4 ;(0.5,0.7)]\right\rangle,\right. \\
&\left\langle x_{2},[(0.2,0.3) ; 0.4 ;(0.5,0.6)]\right\rangle, \\
&\left\langle x_{3},[(0.5,0.6) ; 0.6 ;(0.7,0.8)]\right\rangle, \\
&\left\langle x_{4},[(0.4,0.5) ; 0.5 ;(0.6,0.7)]\right\rangle, \\
&\left\langle x_{5},[(0.2,0.3) ; 0.3 ;(0.4,0.5)]\right\rangle, \\
&\left.\left\langle x_{6},[(0.1,0.2) ; 0.2 ;(0.3,0.4)]\right\rangle\right\}
\end{aligned}
$$

Definition 17. Assuming $(F, A)$ and $(G, B)$ are interval-valued triangular fuzzy soft sets over universe $U$, we say that $(F, A) \wedge$ $(G, B)$ is the "AND" operation on the two sets and $(F, A) \wedge$ $(G, B)=(H, A \times B)$. For $\forall(\alpha, \beta) \in A \times B$, we have

$$
\begin{aligned}
H(\alpha, \beta) & =F(\alpha) \cap G(\beta) \\
& =\left\{\left\langle x, s_{F(\alpha)}(x) \cap s_{G(\beta)}(x)\right\rangle \mid x \in U\right\},
\end{aligned}
$$

where $s_{F(\alpha)}(x)$ and $s_{G(\beta)}(x)$ are the corresponding intervalvalued triangular fuzzy numbers of $x$ in $F(\alpha)$ and $G(\beta)$, respectively.

Definition 18. Assuming $(F, A)$ and $(G, B)$ are interval-valued triangular fuzzy soft sets over universe $U$, we say that
$(F, A) \vee(G, B)$ is the "OR" operation on the two sets, and $(F, A) \vee(G, B)=(M, A \times B)$. For $\forall(\alpha, \beta) \in A \times B$, we have

$$
\begin{aligned}
M(\alpha, \beta) & =F(\alpha) \cup G(\beta) \\
& =\left\{\left\langle x, s_{F(\alpha)}(x) \cup s_{G(\beta)}(x)\right\rangle \mid x \in U\right\},
\end{aligned}
$$

where $s_{F(\alpha)}(x)$ and $s_{G(\beta)}(x)$ are the corresponding intervalvalued triangular fuzzy numbers of $x$ in $F(\alpha)$ and $G(\beta)$, respectively.

Theorem 19. Assuming $(F, A)$ and $(G, B)$ are interval-valued triangular fuzzy soft sets over universe $U$, then we have

$$
\begin{aligned}
& \text { (1) }((F, A) \wedge(G, B))^{c}=(F, A)^{c} \vee(G, B)^{c}, \\
& \text { (2) }((F, A) \vee(G, B))^{c}=(F, A)^{c} \wedge(G, B)^{c} .
\end{aligned}
$$

Proof. (1) and (2) have similar proof, so only (1) is proven in detail. Suppose

$(F, A) \wedge(G, B)=(H, A \times B)$, where

$$
\begin{gathered}
((F, A) \wedge(G, B))^{c}=(H, A \times B)^{c}=\left(H^{c}, A \times B\right), \\
(F, A)^{c} \vee(G, B)^{c}=\left(F^{c}, A\right) \vee\left(G^{c}, B\right)=(M, A \times B) .
\end{gathered}
$$

For $\forall(\alpha, \beta) \in A \times B$, we have

$$
\begin{aligned}
H^{c}(\alpha, \beta) & =(H(\alpha, \beta))^{c} \\
& =\left(\left\{\left\langle x, s_{F(\alpha)}(x) \cap s_{G(\beta)}(x)\right\rangle \mid x \in U\right\}\right)^{c} \\
& =\left\{\left\langle x, s_{F(\alpha)}^{c}(x) \cup s_{G(\beta)}^{c}(x)\right\rangle \mid x \in U\right\} \\
& =F^{c}(\alpha) \cup G^{c}(\beta)=M(\alpha, \beta) .
\end{aligned}
$$

Therefore, $((F, A) \wedge(G, B))^{c}=(F, A)^{c} \vee(G, B)^{c}$ is proven. 
Theorem 20. Assuming $(F, A),(G, B)$, and $(L, C)$ are intervalvalued triangular fuzzy soft sets over universe $U$, then we have

(1) $((F, A) \wedge(G, B)) \wedge(L, C)=(F, A) \wedge((G, B) \wedge(L, C))$,

(2) $((F, A) \vee(G, B)) \vee(L, C)=(F, A) \vee((G, B) \vee(L, C))$.

Proof. Because (1) and (2) have similar proof, only (1) is proven in detail. Suppose

$$
\begin{gathered}
(F, A) \wedge(G, B)=(H, A \times B), \\
(H, A \times B) \wedge(L, C)=(M,(A \times B) \times C), \\
(G, B) \wedge(L, C)=(T, B \times C), \\
(F, A) \wedge(T, B \times C)=(Q, A \times(B \times C)) .
\end{gathered}
$$

For $\forall(\alpha, \beta) \in A \times B, \gamma \in C$, we have

$$
\begin{gathered}
H(\alpha, \beta)=\left\{\left\langle x, s_{F(\alpha)}(x) \cap s_{G(\beta)}(x)\right\rangle \mid x \in U\right\}, \\
M((\alpha, \beta), \gamma) \\
=\left\{\left\langle x,\left(s_{F(\alpha)}(x) \cap s_{G(\beta)}(x)\right) \cap s_{L(\gamma)}(x)\right\rangle \mid x \in U\right\},
\end{gathered}
$$

where $s_{F(\alpha)}(x), s_{G(\beta)}(x)$, and $s_{L(\gamma)}(x)$ are the corresponding interval-valued triangular fuzzy numbers of $x$ in $F(\alpha), G(\beta)$, and $L(\gamma)$, respectively.

For $\forall(\beta, \gamma) \in B \times C, \forall \alpha \in A$, we have

$$
\begin{gathered}
T(\beta, \gamma)=\left\{\left\langle x, s_{G(\beta)}(x) \cap s_{L(\gamma)}(x)\right\rangle \mid x \in U\right\}, \\
Q(\alpha,(\beta, \gamma)) \\
=\left\{\left\langle x, s_{F(\alpha)}(x) \cap\left(s_{G(\beta)}(x) \cap s_{L(\gamma)}(x)\right)\right\rangle \mid x \in U\right\} .
\end{gathered}
$$

Therefore, $((F, A) \wedge(G, B)) \wedge(L, C)=(F, A) \wedge((G, B) \wedge$ $(L, C))$ is established.

Definition 21. Assuming $(F, A)$ and $(G, B)$ are interval-valued triangular fuzzy soft sets over universe $U$, we say that $(F, A) \cap$ $(G, B)$ is the interaction operation on the two sets and $(F, A) \cap$ $(G, B)=(H, C)$, where $C=A \cap B$. For $\forall \varepsilon \in C$, we have

$$
H(\varepsilon)=F(\varepsilon) \cap G(\varepsilon)=\left\{\left\langle x, s_{F(\varepsilon)}(x) \cap s_{G(\varepsilon)}(x)\right\rangle \mid x \in U\right\},
$$

where $s_{F(\varepsilon)}(x)$ and $s_{G(\varepsilon)}(x)$ are the corresponding intervalvalued triangular fuzzy numbers of $x$ in $F(\varepsilon)$ and $G(\varepsilon)$, respectively.

Definition 22. Assuming $(F, A)$ and $(G, B)$ are interval-valued triangular fuzzy soft sets over universe $U$, we say that $(F, A) \cup$ $(G, B)$ is the union operation on the two sets and $(F, A) \cup$ $(G, B)=(M, D)$, where $D=A \cup B$. For $\forall \varepsilon \in D$, we have

$$
M(\varepsilon)=F(\varepsilon) \cup G(\varepsilon)=\left\{\left\langle x, s_{M(\varepsilon)}(x)\right\rangle \mid x \in U\right\} .
$$

Here,

$$
s_{M(\varepsilon)}(x)= \begin{cases}s_{F(\varepsilon)}(x), & \varepsilon \in A-B \\ s_{G(\varepsilon)}(x), & \varepsilon \in B-A \\ s_{F(\varepsilon)}(x) \cup s_{G(\varepsilon)}(x), & \varepsilon \in A \cap B,\end{cases}
$$

where $s_{F(\varepsilon)}(x)$ and $s_{G(\varepsilon)}(x)$ are the corresponding intervalvalued triangular fuzzy numbers of $x$ in $F(\varepsilon)$ and $G(\varepsilon)$, respectively.

Theorem 23. Assuming $(F, A)$ and $(G, B)$ are interval-valued triangular fuzzy soft sets over universe $U$, then we have

$$
\begin{aligned}
& \text { (1) }((F, A) \cup(G, B))^{c}=(F, A)^{c} \cap(G, B)^{c}, \\
& \text { (2) }((F, A) \cap(G, B))^{c}=(F, A)^{c} \cup(G, B)^{c} .
\end{aligned}
$$

Proof. (1) and (2) have similar proof, so only (1) is proven in detail. Suppose $(F, A) \cup(G, B)=(M, D)$, where $((F, A) \cup$ $(G, B))^{c}=(M, D)^{c}=\left(M^{c}, D\right)$, and $(F, A)^{c} \cap(G, B)^{c}=$ $\left(F^{c}, A\right) \cap\left(G^{c}, B\right)=(H, C)$.

For $\forall \varepsilon \in D$, we have

$$
\begin{aligned}
M^{c}(\varepsilon) & =(M(\varepsilon))^{c}=\left(\left\{\left\langle x, s_{M(\varepsilon)}(x)\right\rangle \mid x \in U\right\}\right)^{c} \\
& =\left(\left\{\left\langle x, s_{M(\varepsilon)}^{c}(x)\right\rangle \mid x \in U\right\}\right) \\
& =\left\{\left\langle x, s_{F(\varepsilon)}^{c}(x) \cap s_{G(\varepsilon)}^{c}(x)\right\rangle \mid x \in U\right\} \\
& =F^{c}(\varepsilon) \cap G^{c}(\varepsilon)=H(\varepsilon) .
\end{aligned}
$$

Therefore, $((F, A) \cup(G, B))^{c}=(F, A)^{c} \cap(G, B)^{c}$ is proven.

Theorem 24. Assuming $(F, A),(G, B)$, and $(L, C)$ are intervalvalued triangular fuzzy soft sets over universe $U$, then we have

$$
\begin{aligned}
& (1)((F, A) \cup(G, B)) \cup(L, C)=(F, A) \cup((G, B) \cup(L, C)), \\
& (2)((F, A) \cap(G, B)) \cap(L, C)=(F, A) \cap((G, B) \cap(L, C)) .
\end{aligned}
$$

Proof. (1) and (2) have similar proof, so only (1) is proven in detail. Suppose

$$
\begin{gathered}
(F, A) \cup(G, B)=\left(M_{1}, D_{1}\right), \\
\left(M_{1}, D_{1}\right) \cup(L, C)=\left(M_{2}, D_{2}\right), \\
(G, B) \cup(L, C)=\left(M_{3}, D_{3}\right), \\
(F, A) \cup\left(M_{3}, D_{3}\right)=\left(M_{4}, D_{4}\right),
\end{gathered}
$$

where $D_{2}=D_{4}=A \cup B \cup C$. For $\forall \varepsilon \in D_{1}$, we have $M_{1}(\varepsilon)=$ $\left\{\left\langle x, s_{M_{1}(\varepsilon)}(x)\right\rangle \mid x \in U\right\}$,

$$
s_{M_{1}(\varepsilon)}(x)= \begin{cases}s_{F(\varepsilon)}(x), & \varepsilon \in A-B \\ s_{G(\varepsilon)}(x), & \varepsilon \in B-A \\ s_{F(\varepsilon)}(x) \cup s_{G(\varepsilon)}(x), & \varepsilon \in A \cap B .\end{cases}
$$


For $\forall \varepsilon \in D_{3}$, we have $M_{3}(\varepsilon)=\left\{\left\langle x, s_{M_{3}(\varepsilon)}(x)\right\rangle \mid x \in U\right\}$,

$$
s_{M_{3}(\varepsilon)}(x)= \begin{cases}s_{G(\varepsilon)}(x), & \varepsilon \in B-C \\ s_{L(\varepsilon)}(x), & \varepsilon \in C-B \\ s_{G(\varepsilon)}(x) \cup s_{L(\varepsilon)}(x), & \varepsilon \in B \cap C .\end{cases}
$$

For $\forall \varepsilon \in D_{2}$, we have $M_{2}(\varepsilon)=\left\{\left\langle x, s_{M_{2}(\varepsilon)}(x)\right\rangle \mid x \in U\right\}$,

$$
\begin{aligned}
& s_{M_{2}(\varepsilon)}(x) \\
& = \begin{cases}s_{M_{1}(\varepsilon)}(x), & \varepsilon \in D_{1}-C \\
s_{L(\varepsilon)}(x), & \varepsilon \in C-D_{1} \\
s_{M_{1}(\varepsilon)}(x) \cup s_{L(\varepsilon)}(x), & \varepsilon \in D_{1} \cap C\end{cases} \\
& = \begin{cases}s_{F(\varepsilon)}(x), & \varepsilon \in A-B-C \\
s_{G(\varepsilon)}(x), & \varepsilon \in B-A-C \\
s_{F(\varepsilon)}(x) \cup s_{G(\varepsilon)}(x), & \varepsilon \in A \cap B-C \\
s_{L(\varepsilon)}(x), & \varepsilon \in C-(A \cup B) \\
s_{F(\varepsilon)}(x) \cup s_{L(\varepsilon)}(x), & \varepsilon \in(A-B) \cap C \\
s_{G(\varepsilon)}(x) \cup s_{L(\varepsilon)}(x), & \varepsilon \in(B-A) \cap C \\
s_{F(\varepsilon)}(x) \cup s_{G(\varepsilon)}(x) \cup s_{L(\varepsilon)}(x), & \varepsilon \in B \cap A \cap C .\end{cases}
\end{aligned}
$$

For $\forall \varepsilon \in D_{4}$, we have $M_{4}(\varepsilon)=\left\{\left\langle x, s_{M_{4}(\varepsilon)}(x)\right\rangle \mid x \in U\right\}$,

$$
\begin{aligned}
s_{M_{4}(\varepsilon)}(x) & \begin{cases}s_{F(\varepsilon)}(x), & \varepsilon \in A-D_{3} \\
s_{M_{3}(\varepsilon)}(x), & \varepsilon \in D_{3}-A \\
s_{F(\varepsilon)}(x) \cup s_{M_{3}(\varepsilon)}(x), & \varepsilon \in A \cap D_{3}\end{cases} \\
= & \begin{cases}s_{F(\varepsilon)}(x), & \varepsilon \in A-(B \cup C) \\
s_{L(\varepsilon)}(x), & \varepsilon \in C-B-A \\
s_{G(\varepsilon)}(x), & \varepsilon \in B \cap C-A \\
s_{G(\varepsilon)}(x) \cup s_{L(\varepsilon)}(x), & \varepsilon \in A \cap(B-C) \\
s_{F(\varepsilon)}(x) \cup s_{G(\varepsilon)}(x), & \varepsilon \in A \cap(C-B) \\
s_{F(\varepsilon)}(x) \cup s_{L(\varepsilon)}(x), & \varepsilon \in A \cap B \cap C . \\
s_{F(\varepsilon)}(x) \cup s_{G(\varepsilon)}(x) \cup s_{L(\varepsilon)}(x),\end{cases}
\end{aligned}
$$

From the law of operation among sets, we have $s_{M_{2}(\varepsilon)}(x)=$ $s_{M_{4}(\varepsilon)}(x)$.

Therefore, $((F, A) \cup(G, B)) \cup(L, C)=(F, A) \cup((G, B) \cup$ $(L, C))$ is established.

Theorem 25. Assuming $(F, A),(G, B)$, and $(L, C)$ are intervalvalued triangular fuzzy soft sets over universe $U$, then we have

$$
\begin{aligned}
\text { (1) } & ((F, A) \cup(G, B)) \cap(L, C) \\
& =((F, A) \cap(L, C)) \cup((G, B) \cap(L, C)), \\
(2) & ((F, A) \cap(G, B)) \cup(L, C) \\
& =((F, A) \cup(L, C)) \cap((G, B) \cup(L, C)) .
\end{aligned}
$$

Proof. Only prove (1), because (1) and (2) have similar proof. Assume

$$
\begin{gathered}
(F, A) \cup(G, B)=\left(M_{1}, D_{1}\right), \\
\left(M_{1}, D_{1}\right) \cap(L, C)=\left(H_{1}, D_{2}\right), \\
(F, A) \cap(L, C)=\left(H_{2}, D_{3}\right), \\
(G, B) \cap(L, C)=\left(H_{3}, D_{4}\right), \\
\left(H_{2}, D_{3}\right) \cup\left(H_{3}, D_{4}\right)=\left(M_{2}, D_{5}\right) .
\end{gathered}
$$

Obviously, $D_{2}=D_{5}=(A \cup B) \cap C$. For $\forall \varepsilon \in D_{1}$, we have $M_{1}(\varepsilon)=\left\{\left\langle x, s_{M_{1}(\varepsilon)}(x)\right\rangle \mid x \in U\right\}$,

$$
s_{M_{1}(\varepsilon)}(x)= \begin{cases}s_{F(\varepsilon)}(x), & \varepsilon \in A-B \\ s_{G(\varepsilon)}(x), & \varepsilon \in B-A \\ s_{F(\varepsilon)}(x) \cup s_{G(\varepsilon)}(x), & \varepsilon \in A \cap B .\end{cases}
$$

For $\forall \varepsilon \in D_{2}$, we have

$$
\begin{aligned}
H_{1}(\varepsilon)= & \left\{\left\langle x, s_{H_{1}(\varepsilon)}(x)\right\rangle \mid x \in U\right\} \\
= & \left\{\left\langle x, s_{M_{1}(\varepsilon)}(x) \cap s_{L(\varepsilon)}(x)\right\rangle \mid x \in U\right\}, \\
s_{H_{1}(\varepsilon)}(x) & \\
= & \left\{\begin{array}{cc}
s_{F(\varepsilon)}(x) \cap s_{L(\varepsilon)}(x), & \varepsilon \in(A-B) \cap C \\
s_{G(\varepsilon)}(x) \cap s_{L(\varepsilon)}(x), & \varepsilon \in(B-A) \cap C \\
\left(s_{F(\varepsilon)}(x) \cup s_{G(\varepsilon)}(x)\right) & \\
\cap s_{L(\varepsilon)}(x), & \varepsilon \in(A \cap B) \cap C .
\end{array}\right.
\end{aligned}
$$

For $\forall \varepsilon \in D_{4}$, we have

$$
\begin{aligned}
H_{3}(\varepsilon) & =\left\{\left\langle x, s_{H_{3}(\varepsilon)}(x)\right\rangle \mid x \in U\right\} \\
& =\left\{\left\langle x, s_{G(\varepsilon)}(x) \cap s_{L(\varepsilon)}(x)\right\rangle \mid x \in U\right\} .
\end{aligned}
$$

For $\forall \varepsilon \in D_{5}$, we have

$$
M_{2}(\varepsilon)=\left\{\left\langle x, s_{M_{2}(\varepsilon)}(x)\right\rangle \mid x \in U\right\}
$$




$$
\begin{aligned}
& s_{M_{2}(\varepsilon)}(x) \\
& = \begin{cases}s_{H_{2}(\varepsilon)}(x), & \varepsilon \in D_{3}-D_{4} \\
s_{H_{3}(\varepsilon)}(x), & \varepsilon \in D_{4}-D_{3} \\
s_{H_{2}(\varepsilon)}(x) \cup s_{H_{3}(\varepsilon)}(x), & \varepsilon \in D_{3} \cap D_{4}\end{cases} \\
& = \begin{cases}s_{F(\varepsilon)}(x) \cap s_{L(\varepsilon)}(x), & \varepsilon \in(A \cap C)-(B \cap C) \\
s_{G(\varepsilon)}(x) \cap s_{L(\varepsilon)}(x), & \varepsilon \in(B \cap C)-(A \cap C) \\
\left(s_{F(\varepsilon)}(x) \cap s_{L(\varepsilon)}(x)\right) & \varepsilon \in(B \cap C) \cap(A \cap C) \\
\cup\left(s_{G(\varepsilon)}(x) \cap s_{L(\varepsilon)}(x)\right), & \varepsilon \in(A-B) \cap C\end{cases} \\
& = \begin{cases}s_{F(\varepsilon)}(x) \cap s_{L(\varepsilon)}(x), & \varepsilon \in(B-A) \cap C \\
s_{G(\varepsilon)}(x) \cap s_{L(\varepsilon)}(x), & \varepsilon \in A \cap B \cap C . \\
\left(s_{F(\varepsilon)}(x) \cup s_{G(\varepsilon)}(x)\right) \cap s_{L(\varepsilon)}(x), & \end{cases}
\end{aligned}
$$

Then, $s_{H_{1}(\varepsilon)}(x)=s_{M_{2}(\varepsilon)}(x)$.

Therefore, $((F, A) \cup(G, B)) \cap(L, C)=((F, A) \cap(L, C)) \cup$ $((G, B) \cap(L, C))$ is established.

\section{Decision Making Method of Interval-Valued Triangle Fuzzy Soft Set}

4.1. Determination of Time Weight. In practical decision making problems, the decision-maker masters different information at different time. Normally, the nearer the final moment of the decision is, the more information the decision-maker masters. Thus, they have greater influence on the final decision. While the farther the final moment of the decision is, the less information the decision-maker masters. Thus they have less influence on the final decision. That is to say, the information the decision-maker masters increases with time; accordingly, the effect on the final decision decays forward with time. The effect of different time information on the final decision can be measured in weight, so the problem is turned into how to determine the weight of different times. Reference [33] introduced the weighting methods of basic unit-interval monotonic (BUM) function model, normal distribution model, exponential distribution model, and so forth. Therefore, the weights determination method of exponential decay model can be built on the basis of previous studies.

Theoretically, time $T$ can be divided into continuous and discrete types described, respectively, as follows.

(1) If $T$ is discrete time, $T=\left\{t_{1}, t_{2}, \ldots, t_{N}\right\}$ is denoted. The weight of moment $t_{k}$ is $w_{k}$; thus, $w\left(t_{k}\right)=C_{0} e^{\lambda\left(t_{k}-t_{N}\right)}$, where $C_{0}>0$ is constant and $0<\lambda<1$ is attenuation coefficient.

The weight $w_{k}$ should satisfy $\sum_{k=1}^{N} w\left(t_{k}\right)=\sum_{k=1}^{N}$ $C_{0} e^{\lambda\left(t_{k}-t_{N}\right)}=1$, and we can work out $C_{0}=1 / \sum_{K=1}^{N} e^{\lambda\left(t_{k}-t_{N}\right)}$. Thus,

$$
w\left(t_{k}\right)=\frac{e^{\lambda\left(t_{k}-t_{N}\right)}}{\sum_{k=1}^{N} e^{\lambda\left(t_{k}-t_{N}\right)}}=\frac{e^{\lambda t_{k}}}{\sum_{k=1}^{N} e^{\lambda t_{k}}}, \quad k=1,2, \ldots, N
$$

Particularly, $T=\{1,2, \ldots, N\}, t \in T$; thus weight of time $t$ is

$$
w_{t}=\frac{e^{\lambda t}\left(1-e^{\lambda}\right)}{e^{\lambda}\left(1-e^{\lambda N}\right)}
$$

(2) If $T$ is continuous time, $T=[0, T]$ is denoted. The weight of time $t$ is $w_{k}$; thus, $w(t)=C_{0} e^{\lambda(t-T)}$, where $C_{0}>0$ is constant and $0<\lambda<1$ is attenuation coefficient.

The weight $w_{k}$ should satisfy $\int_{0}^{T} w(t) d t=\int_{0}^{T} C_{0} e^{\lambda(t-T)}$ $d t=1$; we can work out $C_{0}=\lambda /\left(1-e^{-\lambda T}\right)$. Thus we have

$$
w(t)=\frac{\lambda e^{\lambda(t-T)}}{1-e^{-\lambda T}}, \quad T=[0, T] .
$$

Attenuation coefficient $\lambda$ in the model has important practical significance; it reflects accumulative rate of decision-maker's amount of information over time. If accumulative rate at the final moment is higher, it indicates that information which is nearer to the final moment has more effect on the final decision, and the attenuation coefficient is larger; otherwise, the coefficient is smaller. So, decisionmaker can determine the value of attenuation coefficient according to the change of information at different times. This weight determining method can be more flexibly applied to practice.

4.2. Integrated Method of Interval-Valued Triangle Fuzzy Soft Set. The integrated thought in [34] is used to similarly define the arithmetic weighted average operator of interval-valued triangle fuzzy soft set. Then interval-valued triangle fuzzy soft sets of different time are integrated into comprehensive interval-valued triangle fuzzy soft set. According to [27], fuzzy soft set and fuzzy soft matrix are one to one correspondence, so the discussion of the integrated method of intervalvalued triangle fuzzy soft set is turned into the research of the integrated interval-valued triangle fuzzy soft matrix.

Let $U=\left\{x_{1}, x_{2}, \ldots, x_{m}\right\}$ denote the domain and let $A=$ $\left\{e_{1}, e_{2}, \ldots, e_{n}\right\}$ denote the parameter set; the matrix

$$
\begin{array}{r}
\widetilde{F}_{k}=\left(s_{F_{k}\left(e_{j}\right)}\left(x_{i}\right)\right)_{m \times n}, \quad i=1,2, \ldots, m ; \\
j=1,2, \ldots, n ; \quad k=1,2, \ldots, N
\end{array}
$$

is corresponding with interval-valued triangle fuzzy set $\left(F_{k}\right.$, $A$ ), where $s_{F_{k}\left(e_{j}\right)}\left(x_{i}\right)$ is the interval-valued triangle fuzzy number that is corresponding with $x_{i}$ in $F_{k}\left(e_{j}\right)$; the operation rules of interval-valued triangle fuzzy soft matrix are defined as follows.

Definition 26. Suppose $\widetilde{F}_{1}=\left(s_{F_{1}\left(e_{j}\right)}\left(x_{i}\right)\right)_{m \times n}$ and $\widetilde{F}_{2}=\left(s_{F_{2}\left(e_{j}\right)}\right.$ $\left.\left(x_{i}\right)\right)_{m \times n}$ are two interval-valued triangle fuzzy soft matrices, constant $c>0$; then

$$
\begin{aligned}
& \text { (1) } \widetilde{F}_{1}+\widetilde{F}_{2}=\left(s_{F_{1}\left(e_{j}\right)}\left(x_{i}\right)+s_{F_{2}\left(e_{j}\right)}\left(x_{i}\right)\right)_{m \times n}, \\
& \text { (2) } c \widetilde{F}_{1}=\left(c s_{F_{1}\left(e_{j}\right)}\left(x_{i}\right)\right)_{m \times n} .
\end{aligned}
$$


Definition 27. Suppose $\widetilde{F}_{k}(k=1,2, \ldots, N)$ is an intervalvalued triangle fuzzy soft matrix, and the weight satisfies $w_{k} \epsilon$ $[0,1]$ and $\sum_{k=1}^{N} w_{k}=1$; then the arithmetic weighted average operator of interval-valued triangle fuzzy soft set is

$$
f_{w}\left(\widetilde{F}_{1}, \widetilde{F}_{2}, \ldots, \widetilde{F}_{N}\right)=\sum_{k=1}^{N} w_{k} \widetilde{F}_{k} .
$$

Theorem 28. Suppose $\widetilde{F}_{k}(k=1,2, \ldots, K)$ is an intervalvalued triangle fuzzy soft matrix, and the weight satisfies $w_{k} \in$ $[0,1]$ and $\sum_{k=1}^{N} w_{k}=1 . s_{F_{k}\left(\varepsilon_{j}\right)}\left(x_{i}\right)=\left[\left(a_{k i j}^{-}, a_{k i j}^{+}\right) ; b_{k i j} ;\left(c_{k i j}^{-}, c_{k i j}^{+}\right)\right]$ is the interval-valued triangle fuzzy number which is corresponding with $x_{i}$ in $F_{k}\left(\varepsilon_{j}\right)$; then

$$
\begin{aligned}
& f_{w}\left(\widetilde{F}_{1}, \widetilde{F}_{2}, \ldots, \widetilde{F}_{N}\right)=\left(\left[\left(a_{i j}^{-}, a_{i j}^{+}\right) ; b_{i j} ;\left(c_{i j}^{-}, c_{i j}^{+}\right)\right]\right)_{m \times n}, \\
& i=1,2, \ldots, m ; \quad j=1,2, \ldots, n ; \quad k=1,2, \ldots, N,
\end{aligned}
$$

where

$$
\begin{gathered}
a_{i j}^{-}=1-\prod_{k=1}^{N}\left(1-a_{k i j}^{-}\right)^{w_{k}}, \quad a_{i j}^{+}=1-\prod_{k=1}^{N}\left(1-a_{k i j}^{+}\right)^{w_{k}}, \\
b_{i j}=1-\prod_{k=1}^{N}\left(1-b_{k i j}\right)^{w_{k}}, \quad c_{i j}^{-}=1-\prod_{k=1}^{N}\left(1-c_{k i j}^{-}\right)^{w_{k}}, \\
c_{i j}^{+}=1-\prod_{k=1}^{N}\left(1-c_{k i j}^{+}\right)^{w_{k}} .
\end{gathered}
$$

Proof. Use mathematical induction to prove that, when $k=2$, there are

$$
w_{1} \widetilde{F}_{1}=\left(w_{1} s_{F_{1}\left(e_{j}\right)}\left(x_{i}\right)\right)_{m \times n}, \quad w_{2} \widetilde{F}_{2}=\left(w_{2} s_{F_{2}\left(e_{j}\right)}\left(x_{i}\right)\right)_{m \times n} .
$$

Then $w_{1} \widetilde{F}_{1}+w_{2} \widetilde{F}_{2}=\left(w_{1} s_{F_{1}\left(e_{j}\right)}\left(x_{i}\right)+w_{2} s_{F_{2}\left(e_{j}\right)}\left(x_{i}\right)\right)_{m \times n}=$ $\left(\left[\left(a_{i j}^{-}, a_{i j}^{+}\right) ; b_{i j} ;\left(c_{i j}^{-}, c_{i j}^{+}\right)\right]\right)_{m \times n}$, where

$$
\begin{aligned}
a_{i j}^{-}= & \left(1-\left(1-a_{1 i j}^{-}\right)^{w_{1}}\right)+\left(1-\left(1-a_{2 i j}^{-}\right)^{w_{2}}\right) \\
& -\left(1-\left(1-a_{1 i j}^{-}\right)^{w_{1}}\right)\left(1-\left(1-a_{2 i j}^{-}\right)^{w_{2}}\right) \\
= & 1-\left(1-a_{1 i j}^{-}\right)^{w_{1}}\left(1-a_{2 i j}^{-}\right)^{w_{2}} .
\end{aligned}
$$

Similarly,

$$
\begin{aligned}
& a_{i j}^{+}=1-\left(1-a_{1 i j}^{+}\right)^{w_{1}}\left(1-a_{2 i j}^{+}\right)^{w_{2}}, \\
& b_{i j}=1-\left(1-b_{1 i j}\right)^{w_{1}}\left(1-b_{2 i j}\right)^{w_{2}}, \\
& c_{i j}^{-}=1-\left(1-c_{1 i j}^{-}\right)^{w_{1}}\left(1-c_{2 i j}^{-}\right)^{w_{2}}, \\
& c_{i j}^{+}=1-\left(1-c_{1 i j}^{+}\right)^{w_{1}}\left(1-c_{2 i j}^{+}\right)^{w_{2}}
\end{aligned}
$$

satisfy formula (46).
Suppose the formula holds up when $k=M$; then when $k=M+1$, we have

$$
\begin{aligned}
a_{i j}^{-}= & \left(1-\prod_{k=1}^{M}\left(1-a_{k i j}^{-}\right)^{w_{k}}\right)+\left(1-\left(1-a_{(M+1) i j}^{-}\right)^{w_{M+1}}\right) \\
& -\left(1-\prod_{k=1}^{M}\left(1-a_{k i j}^{-}\right)^{w_{k}}\right)\left(1-\left(1-a_{(M+1) i j}^{-}\right)^{w_{M+1}}\right) \\
= & 1-\prod_{k=1}^{M+1}\left(1-a_{k i j}^{-}\right)^{w_{k}} .
\end{aligned}
$$

Similarly

$$
\begin{aligned}
& a_{i j}^{+}=1-\prod_{k=1}^{M+1}\left(1-a_{k i j}^{+}\right)^{w_{k}}, \\
& b_{i j}=1-\prod_{k=1}^{M+1}\left(1-b_{k i j}\right)^{w_{k}}, \\
& c_{i j}^{-}=1-\prod_{k=1}^{M+1}\left(1-c_{k i j}^{-}\right)^{w_{k}}, \\
& c_{i j}^{+}=1-\prod_{k=1}^{M+1}\left(1-c_{k i j}^{+}\right)^{w_{k}}
\end{aligned}
$$

are established.

From the above we can know that formula (46) holds up when $k=M+1$, so formula (46) holds up for any positive integer $k \geq 2$.

4.3. Decision Making Method. For simplicity, $\widetilde{R}=\left(r_{i j}\right)_{m \times n}$ denotes the comprehensive interval-valued triangle fuzzy soft matrix integrated from $\widetilde{F}_{k}(k=1,2, \ldots, N)$, where

$$
\begin{array}{rr}
r_{i j}=\left[\left(a_{i j}^{-}, a_{i j}^{+}\right) ; b_{i j} ;\left(c_{i j}^{-}, c_{i j}^{+}\right)\right], & i=1,2, \ldots, m ; \\
j=1,2, \ldots, n ; \quad k=1,2, \ldots, N .
\end{array}
$$

Both $[8,31]$ use selection values and decision values in the research of the decision problem of interval-valued fuzzy soft set and triangle fuzzy soft set. The paper adopts this thought and gives the selection value $\mu_{i}$ and the decision value $\eta_{i}$ of $x_{i}$ in the domain on the basis of interval-valued triangle fuzzy soft set, where

$$
\begin{aligned}
& \mu_{i}= {\left[\left(a_{i}^{-}, a_{i}^{+}\right) ; b_{i} ;\left(c_{i}^{-}, c_{i}^{+}\right)\right] } \\
&= {\left[\left(\sum_{j=1}^{n} a_{i j}^{-}, \sum_{j=1}^{n} a_{i j}^{+}\right) ; \sum_{j=1}^{n} b_{i j} ;\left(\sum_{j=1}^{n} c_{i j}^{-}, \sum_{j=1}^{n} c_{i j}^{+}\right)\right], } \\
& \eta_{i}=\sum_{t=1}^{m}\left(\left(a_{i}^{-}-a_{t}^{-}\right)+\left(a_{i}^{+}-a_{t}^{+}\right)+\left(b_{i}-b_{t}\right)\right. \\
&\left.+\left(c_{i}^{-}-c_{t}^{-}\right)+\left(c_{i}^{+}-c_{t}^{+}\right)\right) .
\end{aligned}
$$


TABLE 3: Evaluation value of the first year.

\begin{tabular}{llll}
\hline$U$ & $e_{1}$ & $e_{2}$ & $e_{3}$ \\
\hline$x_{1}$ & {$[(0.7,0.8) ; 0.8 ;(0.8,0.9)]$} & {$[(0.5,0.6) ; 0.7 ;(0.7,0.8)]$} & {$[(0.3,0.4) ; 0.5 ;(0.6,0.7)]$} \\
$x_{2}$ & {$[(0.8,0.9) ; 0.9 ;(0.9,1.0)]$} & {$[(0.4,0.5) ; 0.6 ;(0.6,0.7)]$} & {$[(0.4,0.5) ; 0.5 ;(0.5,0.6)]$} \\
$x_{3}$ & {$[(0.4,0.5) ; 0.6 ;(0.6,0.7)]$} & {$[(0.7,0.8) ; 0.8 ;(0.8,0.9)]$} & {$[(0.6,0.7) ; 0.7 ;(0.8,0.9)]$} \\
$x_{4}$ & {$[(0.5,0.6) ; 0.6 ;(0.7,0.8)]$} & {$[(0.7,0.8) ; 0.9 ;(0.9,1.0)]$} & {$[(0.5,0.6) ; 0.6 ;(0.7,0.8)]$} \\
\hline
\end{tabular}

TABLE 4: Evaluation value of the second year.

\begin{tabular}{llll}
\hline$U$ & $e_{1}$ & $e_{2}$ & $e_{3}$ \\
\hline$x_{1}$ & {$[(0.5,0.6) ; 0.6 ;(0.7,0.8)]$} & {$[(0.5,0.6) ; 0.6 ;(0.7,0.8)]$} & {$[(0.4,0.5) ; 0.6 ;(0.6,0.7)]$} \\
$x_{2}$ & {$[(0.7,0.8) ; 0.8 ;(0.8,0.9)]$} & {$[(0.5,0.6) ; 0.6 ;(0.7,0.8)]$} & {$[(0.3,0.4) ; 0.4 ;(0.4,0.5)]$} \\
$x_{3}$ & {$[(0.5,0.6) ; 0.7 ;(0.7,0.8)]$} & {$[(0.6,0.7) ; 0.8 ;(0.8,0.9)]$} & {$[(0.7,0.8) ; 0.8 ;(0.8,0.9)]$} \\
$x_{4}$ & {$[(0.5,0.6) ; 0.7 ;(0.8,0.9)]$} & {$[(0.6,0.7) ; 0.7 ;(0.7,0.8)]$} & {$[(0.4,0.5) ; 0.5 ;(0.5,0.6)]$} \\
\hline
\end{tabular}

TABLE 5: Evaluation value of the third year.

\begin{tabular}{llcc}
\hline$U$ & $e_{1}$ & $e_{2}$ & $e_{3}$ \\
\hline$x_{1}$ & {$[(0.6,0.7) ; 0.8 ;(0.8,0.9)]$} & {$[(0.5,0.6) ; 0.7 ;(0.8,0.9)]$} & {$[(0.5,0.6) ; 0.6 ;(0.7,0.8)]$} \\
$x_{2}$ & {$[(0.5,0.6) ; 0.6 ;(0.6,0.7)]$} & {$[(0.3,0.4) ; 0.5 ;(0.5,0.6)]$} & {$[(0.2,0.3) ; 0.3 ;(0.4,0.5)]$} \\
$x_{3}$ & {$[(0.7,0.8) ; 0.8 ;(0.8,0.9)]$} & {$[(0.7,0.8) ; 0.9 ;(0.9,1.0)]$} & {$[(0.7,0.8) ; 0.8 ;(0.9,1.0)]$} \\
$x_{4}$ & {$[(0.4,0.5) ; 0.6 ;(0.7,0.8)]$} & {$[(0.5,0.6) ; 0.6 ;(0.6,0.7)]$} & {$[(0.3,0.4) ; 0.4 ;(0.5,0.6)]$} \\
\hline
\end{tabular}

TABLE 6: Comprehensive interval-valued triangle fuzzy soft set.

\begin{tabular}{ccccc}
\hline$U$ & $e_{1}$ & $e_{2}$ & $e_{3}$ \\
\hline$x_{1}$ & {$[(0.5940,0.6961) ; 0.7525 ;(0.7735,0.8763)]$} & {$[(0.5000,0.6000) ; 0.6723 ;(0.7557,0.8592)]$} & {$[(0.4370,0.5380) ; 0.5830 ;(0.6542,0.7557)]$} \\
$x_{2}$ & {$[(0.6397,0.7503) ; 0.7503 ;(0.7503,1.0000)]$} & {$[(0.3866,0.4880) ; 0.5521 ;(0.5900,0.6936)]$} & {$[(0.2722,0.3729) ; 0.3729 ;(0.4200,0.5204)]$} \\
$x_{3}$ & {$[(0.6006,0.7065) ; 0.7422 ;(0.7422,0.8482)]$} & {$[(0.6723,0.7735) ; 0.8592 ;(0.8592,1.0000)]$} & {$[(0.6835,0.7843) ; 0.7843 ;(0.8592,1.0000)]$} \\
$x_{4}$ & {$[(0.4516,0.5521) ; 0.6338 ;(0.7351,0.8384)]$} & {$[(0.5755,0.6782) ; 0.7172 ;(0.7172,1.0000)]$} & {$[(0.3729,0.4740) ; 0.4740 ;(0.5454,0.6485)]$} \\
\hline
\end{tabular}

4.4. Decision Procedure. Let $U=\left\{x_{1}, x_{2}, \ldots, x_{m}\right\}$ denote the domain, let $A=\left\{e_{1}, e_{2}, \ldots, e_{n}\right\}$ denote parameter set, let $T=\{1,2, \ldots, N\}$ denote time set, time weight vector is $w=\left(w_{1}, w_{2}, \ldots, w_{N}\right)$, according to the above discussion, and dynamic decision steps can be got on the basis of intervalvalued triangle fuzzy soft set.

Step 1. Determine the attenuation coefficient $\lambda$ according to the information mastered by the decision-maker, and use formula (41) to calculate weights of every moment.

Step 2. Use formula (46) to turn interval-valued triangle fuzzy soft matrices of different moments into comprehensive interval-valued triangle fuzzy soft matrices.

Step 3. Use formulas (54) and (55) to calculate the selection value and decision value of every element in the domain, respectively.

Step 4. Make decision making analysis based on the decision values; the corresponding element of the biggest decision value is the best one.

\section{Example Analysis}

A venture capital firm considers choosing one of the 4 alternative enterprises $\left(x_{1}, x_{2}, x_{3}, x_{4}\right)$ to invest; the investigation period is nearly 3 years; the following three aspects are investigated: economic benefits $\left(e_{1}\right)$, social benefits $\left(e_{2}\right)$, and sustainable development capacity $\left(e_{3}\right)$ of every year. The evaluation results are given in the form of interval-valued triangle fuzzy soft set in Tables 3, 4, and 5.

Let the attenuation coefficient be $\lambda=0.5$; we use formula (41) to calculate the time weight $w=(0.1863,0.3072,0.5065)$. Use formula (46) to turn interval-valued triangle fuzzy soft sets into comprehensive interval-valued triangle fuzzy soft set, as shown in Table 6.

Selection values of each enterprise calculated based on formula (54) are as follows:

$$
\begin{aligned}
& \mu_{1}=[(1.5310,1.8341) ; 2.0078 ;(2.1834,2.4912)], \\
& \mu_{2}=[(1.2985,1.6112) ; 1.6753 ;(1.7603,2.2140)], \\
& \mu_{3}=[(1.9564,2.2643) ; 2.3857 ;(2.4606,2.8482)], \\
& \mu_{4}=[(1.4000,1.7043) ; 1.8250 ;(1.9977,2.4869)] .
\end{aligned}
$$


Decision values of each enterprise calculated based on formula (55) are as follows:

$$
\begin{array}{ll}
\eta_{1}=0.2541, & \eta_{2}=-5.6987, \\
\eta_{3}=7.7249, & \eta_{4}=-2.2803 .
\end{array}
$$

Because $\eta_{3}$ is the biggest value, investing enterprise $x_{3}$ is the best solution.

\section{Conclusion}

In order to improve fuzzy soft set theory, the concept of interval-valued triangle fuzzy soft set is presented; in addition, some relevant operational properties are given and proven. Because the dynamic decision model based on interval-valued triangle fuzzy soft considers the influence of time variation, the decision making process is more realistic, and the decision making result got by integrated operation is more reliable. At last, the practical example analysis shows that the decision making method presented in this paper is effective and that programming is easy to do with it. Besides, it is necessary to point out that the research method by interval-valued triangle fuzzy soft set can be extended to other methods, like the interval-valued trapezoidal fuzzy soft set theory.

\section{Conflict of Interests}

The authors declare that there is no conflict of interests regarding the publication of this paper.

\section{Acknowledgments}

This work is supported by the Natural Science Foundation of China (no. 51105135) and young talents funded projects of Heilongjiang University of Science and Technology (no. 20120501).

\section{References}

[1] Y. Zou and Z. Xiao, "Data analysis approaches of soft sets under incomplete information," Knowledge-Based Systems, vol. 21, no. 8, pp. 941-945, 2008.

[2] L. A. Zadeh, "Fuzzy sets," Information and Control, vol. 8, no. 3, pp. 338-353, 1965.

[3] Z. Pawlak, "Rough sets," International Journal of Computer and Information Sciences, vol. 11, no. 5, pp. 341-356, 1982.

[4] M. B. Gorzałczany, "A method of inference in approximate reasoning based on interval-valued fuzzy sets," Fuzzy Sets and Systems, vol. 21, no. 1, pp. 1-17, 1987.

[5] D. Molodtsov, "Soft set theory: first results," Computers \& Mathematics with Applications, vol. 37, no. 4-5, pp. 19-31, 1999.

[6] P. K. Maji, R. Biswas, and A. R. Roy, "Fuzzy soft sets," Journal of Fuzzy Mathematics, vol. 9, no. 3, pp. 589-602, 2001.

[7] P. K. Maji, R. Biswas, and A. R. Roy, "Intuitionistic fuzzy soft sets," Journal of Fuzzy Mathematics, vol. 9, no. 3, pp. 677-692, 2001.
[8] X. Yang, T. Y. Lin, J. Yang, Y. Li, and D. Yu, "Combination of interval-valued fuzzy set and soft set," Computers \& Mathematics with Applications, vol. 58, no. 3, pp. 521-527, 2009.

[9] Y. Jiang, Y. Tang, Q. Chen, H. Liu, and J. Tang, "Interval-valued intuitionistic fuzzy soft sets and their properties," Computers \& Mathematics with Applications, vol. 60, no. 3, pp. 906-918, 2010.

[10] F. Feng, C. Li, B. Davvaz, and M. I. Ali, "Soft sets combined with fuzzy sets and rough sets: a tentative approach," Soft Computing, vol. 14, no. 9, pp. 899-911, 2010.

[11] M. I. Ali, "A note on soft sets, rough soft sets and fuzzy soft sets," Applied Soft Computing Journal, vol. 11, no. 4, pp. 3329-3332, 2011.

[12] W. Xu, J. Ma, S. Wang, and G. Hao, "Vague soft sets and their properties," Computers and Mathematics with Applications, vol. 59, no. 2, pp. 787-794, 2010.

[13] W. Gau and D. J. Buehrer, "Vague sets," IEEE Transactions on Systems, Man and Cybernetics, vol. 23, no. 2, pp. 610-614, 1993.

[14] P. Majumdar and S. K. Samanta, "Generalised fuzzy soft sets," Computers \& Mathematics with Applications, vol. 59, no. 4, pp. 1425-1432, 2010.

[15] M. I. Ali, F. Feng, X. Liu, W. K. Min, and M. Shabir, "On some new operations in soft set theory," Computers and Mathematics with Applications, vol. 57, no. 9, pp. 1547-1553, 2009.

[16] A. Sezgin and A. O. Atagün, "On operations of soft sets," Computers \& Mathematics with Applications, vol. 61, no. 5, pp. 14571467, 2011.

[17] P. K. Maji, A. R. Roy, and R. Biswas, "On intuitionistic fuzzy soft sets," Journal of Fuzzy Mathematics, vol. 12, no. 3, pp. 669-683, 2004.

[18] Y. B. Jun, K. J. Lee, and C. H. Park, "Soft set theory applied to ideals in d-algebras," Computers \& Mathematics with Applications, vol. 57, no. 3, pp. 367-378, 2009.

[19] Y. B. Jun, K. J. Lee, and J. Zhan, "Soft p-ideals of soft BCI-algebras," Computers and Mathematics with Applications, vol. 58, no. 10, pp. 2060-2068, 2009.

[20] W. K. Min, "Similarity in soft set theory," Applied Mathematics Letters, vol. 25, no. 3, pp. 310-314, 2012.

[21] H. Aktaş and N. Çağman, "Soft sets and soft groups," Information Sciences, vol. 177, no. 13, pp. 2726-2735, 2007.

[22] Z. Xiao, K. Gong, and Y. Zou, "A combined forecasting approach based on fuzzy soft sets," Journal of Computational and Applied Mathematics, vol. 228, no. 1, pp. 326-333, 2009.

[23] P. K. Maji, A. R. Roy, and R. Biswas, "An application of soft sets in a decision making problem," Computers \& Mathematics with Applications, vol. 44, no. 8-9, pp. 1077-1083, 2002.

[24] A. R. Roy and P. K. Maji, "A fuzzy soft set theoretic approach to decision making problems," Journal of Computational and Applied Mathematics, vol. 203, no. 2, pp. 412-418, 2007.

[25] Z. Kong, L. Gao, and L. Wang, "Comment on 'a fuzzy soft set theoretic approach to decision making problems"' Journal of Computational and Applied Mathematics, vol. 223, no. 2, pp. 540-542, 2009.

[26] N. Çağman and S. Enginoğlu, "Soft set theory and uni-int decision making," European Journal of Operational Research, vol. 207, no. 2, pp. 848-855, 2010.

[27] N. Çağman and S. Enginoğ, "Soft matrix theory and its decision making," Computers \& Mathematics with Applications, vol. 59, no. 10, pp. 3308-3314, 2010. 
[28] F. Feng, Y. B. Jun, X. Liu, and L. Li, "An adjustable approach to fuzzy soft set based decision making," Journal of Computational and Applied Mathematics, vol. 234, no. 1, pp. 10-20, 2010.

[29] Y. C. Jiang, Y. Tang, and Q. M. Chen, "An adjustable approach to intuitionistic fuzzy soft sets based decision making," Applied Mathematical Modelling, vol. 35, no. 2, pp. 824-836, 2011.

[30] J. J. Mao, D. B. Yao, and C. C. Wang, "Group decision-making method based on time-series fuzzy soft sets," Systems Engineering Theory \& Practice, vol. 34, no. 1, pp. 182-189, 2014.

[31] T. Kuang and Z. Xiao, "A multi-criteria decision making approach based on triangle-valued fuzzy soft sets," Journal of Convergence Information Technology, vol. 7, no. 15, pp. 17-25, 2012.

[32] T. Kuang, "Trapezoid -valued fuzzy soft sets and its applications," Advances in Information Sciences and Service Sciences, vol. 4, no. 15, pp. 310-316, 2012.

[33] Z. Xu and R. R. Yager, "Dynamic intuitionistic fuzzy multi-attribute decison making," International Journal of Approximate Reasoning, vol. 48, no. 1, pp. 246-262, 2008.

[34] Z. S. Xu, "Intuitionistic fuzzy aggregation operators," IEEE Transactions on Fuzzy Systems, vol. 15, no. 6, pp. 1179-1187, 2007.

[35] S. Zhang, S. Liu, and R. Zhai, "An extended GRA method for MCDM with interval-valued triangular fuzzy assessments and unknown weights," Computers and Industrial Engineering, vol. 61, no. 4, pp. 1336-1341, 2011. 


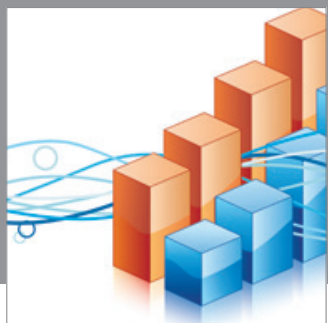

Advances in

Operations Research

mansans

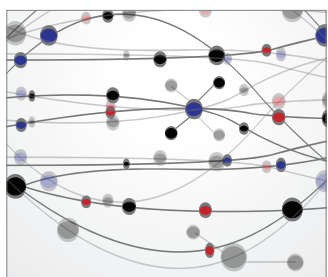

The Scientific World Journal
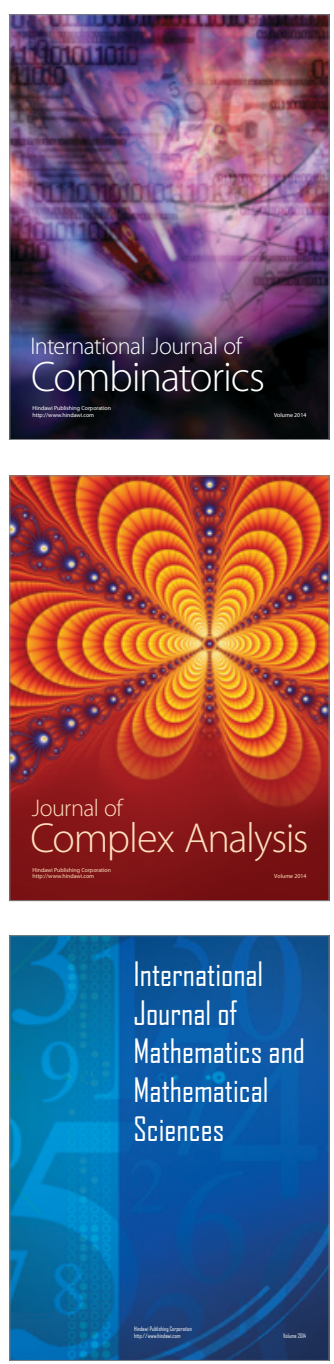
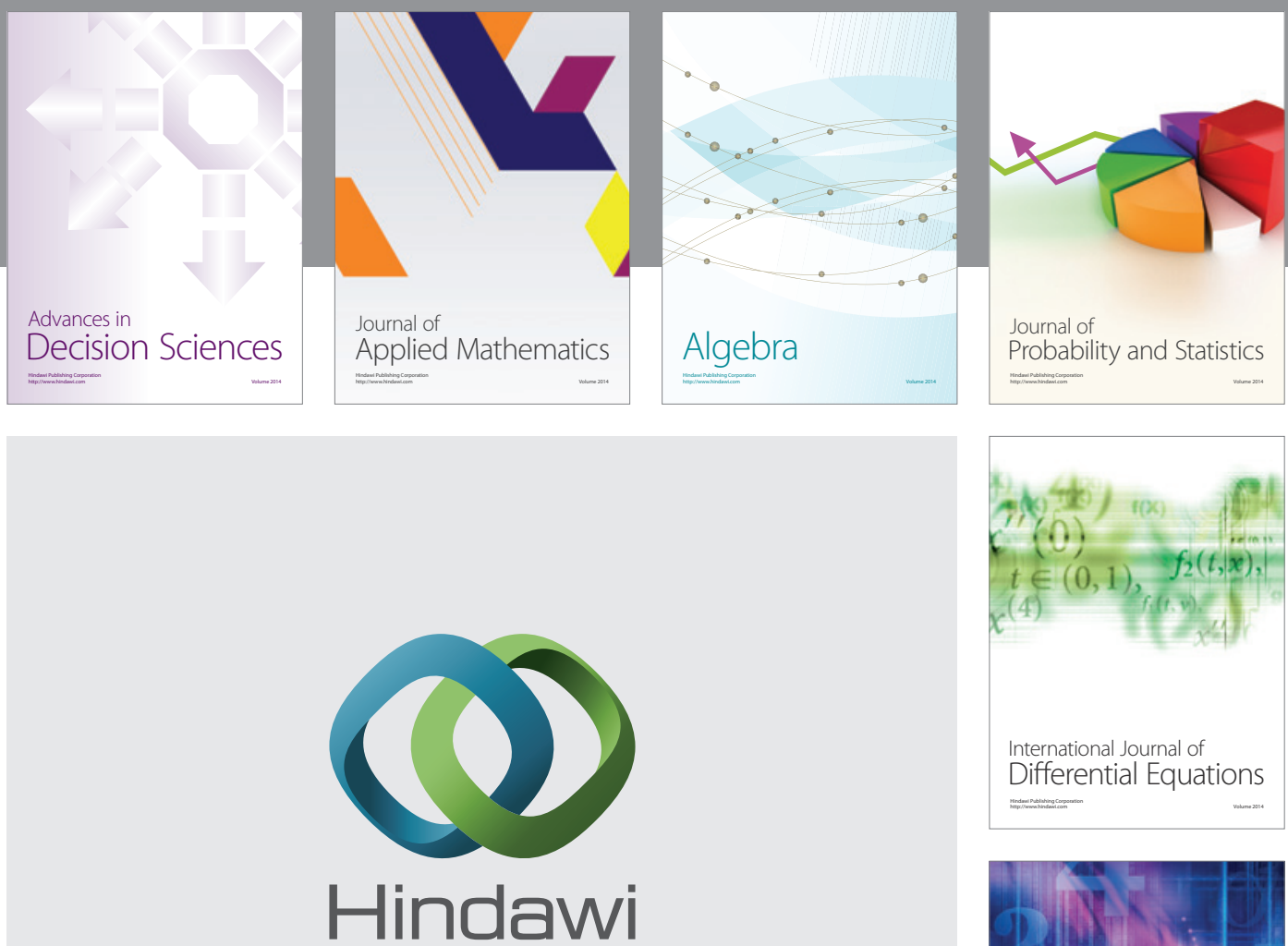

Submit your manuscripts at http://www.hindawi.com
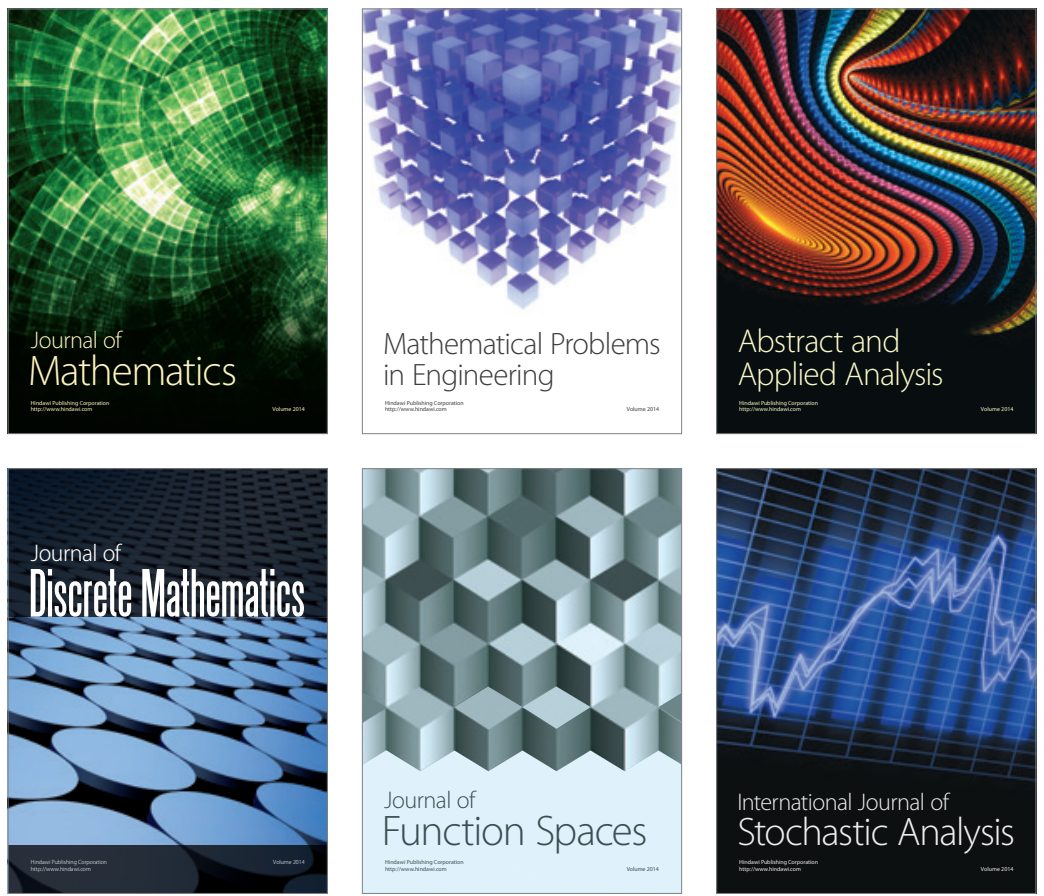

Journal of

Function Spaces

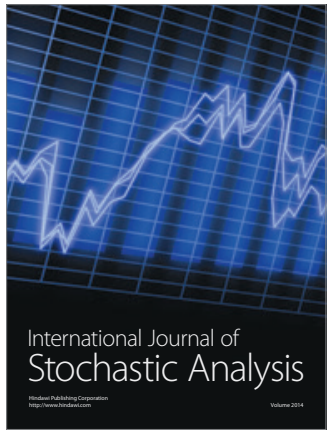

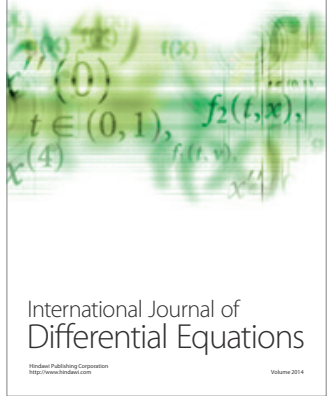
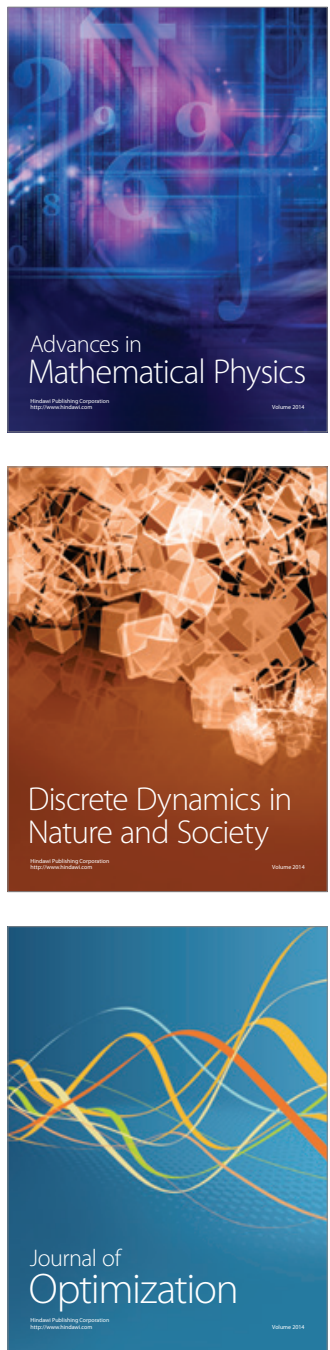